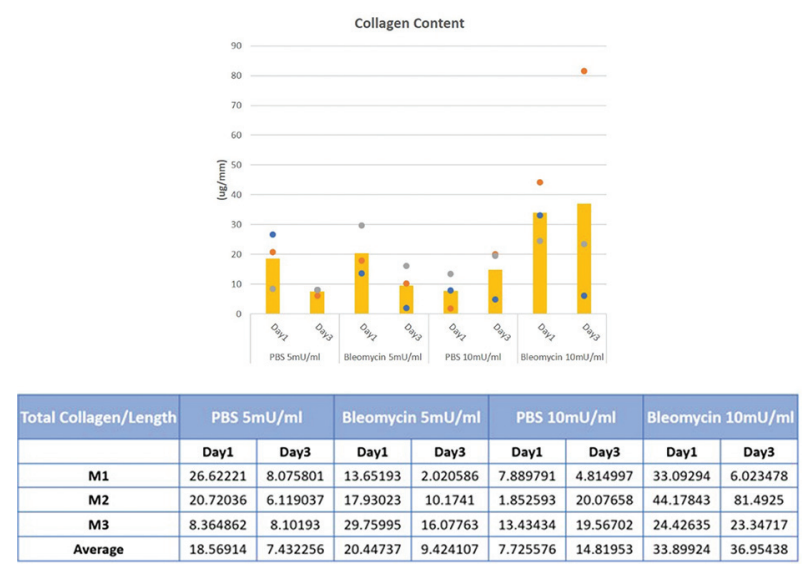

Abstract THU0356 - Figure 2. Total collagen content

ADSC Absolute Cell Count on Day1

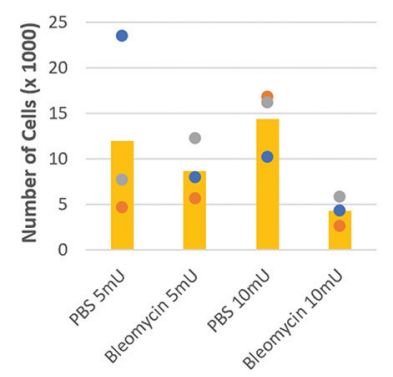

ADSC Absolute Cell Count on Day3
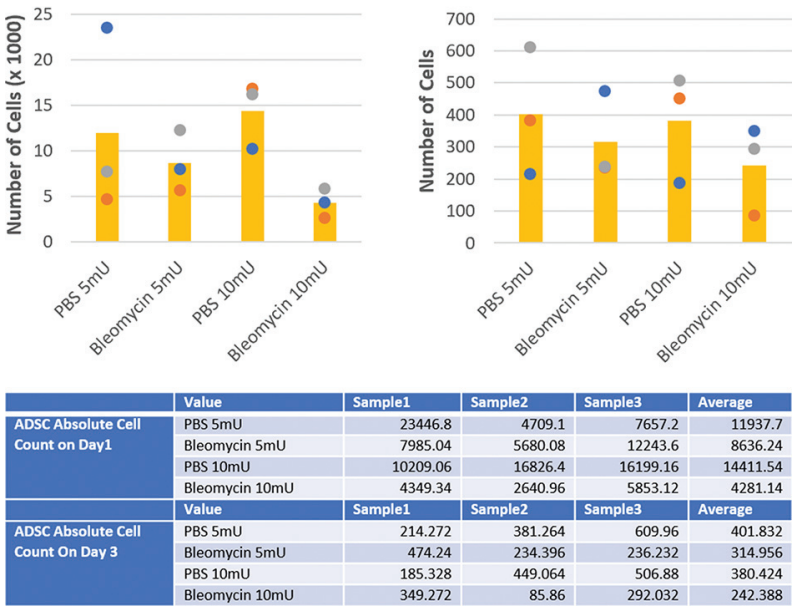

\begin{tabular}{|r|r|r|r|}
\hline mple1 & \multicolumn{1}{|c|}{ Sample2 } & \multicolumn{1}{|c|}{ Sample3 } & \multicolumn{1}{|c|}{ Average } \\
\hline 23446.8 & 4709.1 & 7657.2 & 11937.7 \\
\hline 7985.04 & 5680.08 & 12243.6 & 8636.24 \\
\hline 10209.06 & 16826.4 & 16199.16 & 14411.54 \\
\hline 4349.34 & \multicolumn{1}{|c|}{2640.96} & \multicolumn{1}{|c|}{5853.12} & 4281.14 \\
\hline mple1 & Sample2 & \multicolumn{1}{|c|}{ Sample3 } & Average \\
\hline 214.272 & 381.264 & 609.96 & 401.832 \\
\hline 474.24 & 234.396 & 236.232 & 314.956 \\
\hline 185.328 & 449.064 & 506.88 & 380.424 \\
\hline 349.272 & 85.86 & 292.032 & 242.388 \\
\hline
\end{tabular}

Abstract THU0356 - Figure 3. ADSC cell count measured by flow cytometry

Disclosure of Interests: None declared

DOI: 10.1136/annrheumdis-2019-eular.1881

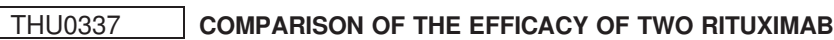 REGIMENS IN THE PATIENTS WITH SYSTEMIC SCLEROSIS ASSOCIATED WITH INTERSTITIAL LUNG DISEASE}

Olga Koneva, Lidia P. Ananyeva, Liudmila Garzanova, Oxana Desinova, Olga Ovsyannikova, Mayya Starovoytova. V.A. Nasonova Research Institute of Rheumatology, Moscow, Russian Federation

Background: Rituximab (RTM) is considered as a promising therapeutic agent for treatment of insterstitial lung disease (ILD) in the patients with systemic sclerosis (SSc). However, the limited number of RTM-treated patients, heterogeneity of the studies in relation to main parameters, considerably different dose regimens, cumulative doses, and observation periods does not allow univocal conclusions on RTM efficacy or definitive recommendations on RTM use in the patients with SSc. The question whether to combine RTM with immunosupressants (IS) or it is possible to use it as a single-agent therapy in the patients with SSc associated with ILD is still relevant.

Objectives: To compare the time courses of pulmonary function parameters and dermal fibrosis parameters during the use of RTM in combination with IS and as a single-agent therapy in the patients with SSc associated with ILD in the open-label prospective non-randomized study. Methods: 90 patients with the confirmed SSc diagnosis and ILD evidence based on HRCT findings were enrolled into the study. All patients received low-dose and moderate-dose prednisolone regimens. Group A $(\mathrm{n}=45)$ received a total $\mathrm{RTM}$ dose $3.1 \pm 1.2 \mathrm{~g}$ in combination with IS
(27/60\% mycophenolate mofetyl, 16/35.6\% cyclophosphamide, 2/4.4\% methotrexate; the patient's average age was $47.4 \pm 11.6$ years, with female proportion $82 \%$; SSc duration $4.6 \pm 3.5$ years; diffused/localized forms $1.3 /$ 1). Group B $(n=45)$ received $R T M$ as a single therapy agent in a total dose $2.7 \pm 1 \mathrm{~g}$ (average age $45.0 \pm 15$ years, female proportion $82 \%$, SSc duration $6.7 \pm 5.6$ years, diffused/localized forms 1.5/1). The age, gender proportion, SSc form, FVC and DLCO, and RTM cumulative doses were similar in the both groups. The follow-up period was 42 months. The time courses of FVC, DLCO, modified skin count (mRss, points), activity index (EScSG, points) were assessed in the study.

Results: In Groups A and B during the therapy significant decrease in mRss ( $p=0.00034$ and 0.000002 respectively) and EScSG $(p=0.00011$ and 0.000000 respectively), FVC increase $(p ;=0.00017$ and 0.00001 , respectively), and stabilization of the DLCO were observed.

The treatment groups did not differ significantly in the median FVC increment, clinically meaningful FVC and DLCO increments of decrements, and EScSG and mRss time courses.

\begin{tabular}{lcc}
\hline Parameters & Group A & Group B \\
\hline EScSG 1 & $3.2 \pm 1.9^{*}$ & $2.7 \pm 1.6^{*}$ \\
$M \pm S D$ & $1.6 \pm 1.3^{*}$ & $1.2 \pm 0.98^{*}$ \\
EScSG 2 & & \\
$M \pm S D$ & $11.0 \pm 9.3^{*}$ & $11.5 \pm 9.3^{*}$ \\
mRss 1 & & \\
$M \pm S D$ & $7.2 \pm 5.6^{*}$ & $5.6 \pm 4.2^{*}$ \\
mRss 2 & & \\
$M \pm S D$ & $76 \pm 20.3^{*}$ & $77.8 \pm 19.7^{*}$ \\
FVC $1 M \pm S D$ & $82.7 \pm 22.5^{*}$ & $86.8 \pm 19.7^{*}$ \\
FVC $2 M \pm S D$ & 5.7 & 8.3 \\
$\Delta$ FVC $\%$ & {$[0 ; 11.2]$} & {$[1.1 ; 15.4]$} \\
& $14 / 31$ & $17 / 37.8$ \\
FVC increment by $\geq 10 \%, \mathrm{n} / \%$ & $2 / 4.4$ & $2 / 4.4$ \\
FVC decrement by $\geq 10 \% \mathrm{n} / \%$ & $44.5 \pm 19$ & $47.8 \pm 17.1$ \\
DLCO $1 M \pm S D$ & $45.4 \pm 16.8$ & $50.4 \pm 16.9$ \\
DLCO $2 M \pm S D$ & 1.3 & 2.1 \\
$\Delta$ DLCO $\%$ & {$[-1.6 ; 6.9]$} & {$[-1.41 ; 7.1]$} \\
DLCO increment by $\geq 10 \%, \mathrm{n} / \%$ & $3 / 6.7$ & $5 / 11$ \\
DLCO decrement by $\geq 10 \% \mathrm{n} / \%$ & $5 / 11$ & $4 / 8.9$ \\
\hline
\end{tabular}

Notes: in Parameters column 1 = before treatment, 2 = after treatment; $M \pm S D=$ mean value and standard deviation; * significant difference between the vales measured before and after the treatment

Conclusion: RTM administration both in combination with IS and as a single agent therapy in the patients with SSc associated with ILD effectively alleviated skin induration and EScSG, improved or stabilized the pulmonary function parameters. The absence of statistically significant difference in the time course of evaluated parameters between the groups substantiate potential RTM use as a single-agent therapy that, this is most important for the patients with poor tolerability or contraindications to IS administration.

Disclosure of Interests: None declared

DOI: 10.1136/annrheumdis-2019-eular.6870

\section{THU0338 SIMVASTATIN-CONJUGATED NANOPARTICLE ENHANCES THE THERAPEUTIC EFFECT OF ADIPOSE- DERIVED STEM CELLS ON INTERSTITIAL LUNG DISEASE}

Takuya Kotani, Takayasu Suzuka, Shogo Matsuda, II Masaaki, Tohru Takeuchi, Shigeki Arawaka. Osaka Medical College, Takatsuki, Japan

Background: Interstitial lung disease (ILD) associated with connective tis sue disease is a life-threatening pathological condition that causes respiratory failure when it progresses. Lung inflammation is treated with corticosteroids and immunosuppressants, and pulmonary fibrosis is treated with anti-fibrosis agents such as pirfenidone and nintedanib. However, many cases are treatment-resistant and the outcome is poor. Moreover adverse effects such as infections resulting from immunosuppressive therapy are problematic. The development of new treatments is thus required for ILD from the viewpoint of the poor effect and adverse effects of the currently available treatments. Research and development with adiposederived stem cells (AdSCs) in immunosuppressive therapy have progressed for autoimmune diseases, and favorable outcomes have been reported. In recent years, the effectiveness of AdSCs in ILD model mice has been demonstrated (ref). The statin preparation has not only an angiogenesis promoting action, an immunosuppressive action, an antifibrotic action but also an action of promoting a cellular function including a migratory ability. 
Objectives: We have investigated the hypothesis that statin, an agent with pleiotropic effects, could augment the therapeutic potential of AdSCs. Methods: ILD was induced by bleomycin (BLM) in C57BL/6 mouse, and the mice were assigned in the following groups: 1) Control, 2) NP-AdSCs $\left(2.5 \times 10^{4}\right.$ cells), and 3$)$ STNP-AdSCs $\left(2.5 \times 10^{4}\right.$ cells $)$.

Results: Simvastatin-conjugated nanoparticles (STNP) significantly promoted the migration activity and cell survival without changing the proliferation activity, and up-regulated transforming growth factor (TGF) $-\beta 1$ in vitro assays. Lung inflammation and fibrosis assessed were significantly supressed at 4 weeks after starting BLM administration in STNP-AdSCs group (Figure). The levels of IL-4, IFN-gamma, TNF-alpha, COL1A1, and TIMP1 mRNA expression at 28 days after BLM administration were significantly lower in STNP-AdSCs group compared with that in other groups. Conclusion: Simvastatin-conjugated nanoparticles enhanced the therapeutic effect of a small number of AdSCs transplantation.
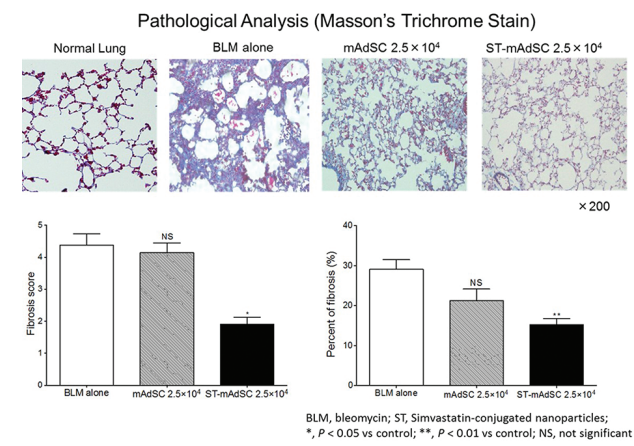

Abstract THU0338 - Figure 1

REFERENCE:

[1] Sci Rep 6; 7 (1): 14608, 2017.

Acknowledgement: This study was supported by Grants-in-Aid for Scientific Research-KAKENHI- of Japan (18K08160).

Disclosure of Interests: None declared

DOI: 10.1136/annrheumdis-2019-eular.6636

\section{THU0339 PULMONARY INVOLVEMENT AND OUTCOME IN SYSTEMIC SCLEROSIS (SSC) - ILD-PH AS AN IMPORTANT SUBSET}

Michael Kreuter ${ }^{1}$, Francesco Bonella ${ }^{2}$, Ulf Müller-Ladner ${ }^{3}$, Elise Siegert ${ }^{4}$ Jörg Henes ${ }^{5}$, Gabriela Riemekasten ${ }^{6}$, Norbert Blank ${ }^{7}$, Marc Schmalzing ${ }^{8}$, Ina Koetter ${ }^{9}$, Claudia Guenther ${ }^{10}$, Tim Schmeiser ${ }^{11}$, Gabriele Zeidler ${ }^{12}$, Alexander Kreuter ${ }^{13}$, Margitta Worm ${ }^{14}$, Laura Susok ${ }^{15}$, Aaron Juche ${ }^{16}$, Christiane Pfeiffer ${ }^{17}$, Cord Sunderkoetter ${ }^{18}$, Donja Homayoon ${ }^{19}$, Kathrin Kuhr ${ }^{20}$, Hanns-Martin Lorenz ${ }^{21}$, Pia Moinzadeh ${ }^{22}$, Nicolas Hunzelmann ${ }^{22} .{ }^{1}$ Center for interstitial and rare lung disease, Thoraxklinik, University Hospital, Pneumology, Heidelberg, Germany; ${ }^{2}$ Center for interstitial and rare lung disease Ruhrlandklinik University Hospital, Essen, Germany; ${ }^{3}$ Justus Liebig University, Campus Kerckhoff, Rheumatology, Giessen, Germany; ${ }^{4}$ Charité - Universitätsmedizin Berlin, Rheumatology, Berlin, Germany, ${ }^{5}$ Center for Interdisciplinary Clinical Immonology, Rheumatology and Autoinflammatory Diseases, University Hospital, Tuebingen, Germany; ${ }^{6}$ University Medical Center - UKSH, Rheumatology, Luebeck, Germany, ${ }^{7}$ University Hospital Heidelberg, Rheumatology, Heidelberg, Germany; ${ }^{8}$ University Hospital Würzburg, Rheumatology, Würzburg, Germany; ${ }^{9}$ Asklepios Clinik Altona, Internal Medicine, Rheumatology, Immunology and Nephrology, Hamburg, Germany, ${ }^{10}$ University Hospital Carl Gustav Carus, Dermatology, Dresden, Germany, ${ }^{11}$ Hospital St. Joseph, Rheumatology, Wuppertal, Germany, ${ }^{12}$ Johanniter-Krankenhaus im Fläming, Rheumatology, Treuenbrietzen, Germany; ${ }^{13} \mathrm{HELIOS}$ St. Elisabeth Hospital Oberhausen, University Witten-Herdecke, Dermatology, Oberhausen, Germany, ${ }^{14}$ Charité Universitätsmedizin Berlin, Dermatology, Berlin, Germany; ${ }^{15}$ Ruhr-UniversityBochum, St. Josef Hospital, Dermatology, Bochum, Germany, ${ }^{16}$ Immanuel Hospital Berlin (Buch), Rheumatology, Berlin, Germany, ${ }^{17}$ University Hospital Ulm, Dermatology, Ulm, Germany, ${ }^{18}$ University Hospital Halle (Saale), Dermatology, Halle, Germany; ${ }^{19}$ Medical University of Graz, Dermatology, Graz, Austria; ${ }^{20}$ University of Cologne, IMSB, Cologne, Germany; ${ }^{21}$ Center for interstitial and rare lung disease Ruhrlandklinik University Hospital, Pneumology, Essen, Germany; ${ }^{22}$ University Hospital Cologne, Dermatology, Cologne, Germany

Background: Pulmonary involvement is the leading cause of death in SSc and can manifest as interstitial lung disease (ILD), pulmonary hypertension (PAH) or a combination (ILD-PH). Aim of this analysis was to determine prevalence, clinical characteristics and outcomes of different forms within the German SSc Network.

Objectives:

Methods: SSc patients were analyzed for pulmonary involvement, clinical characteristics and outcome.

Results: There were 3699 pts in 42 centers with a mean follow up time of $34.4 \pm 12.6$ months. At baseline, ILD was frequent $(29.5 \%)$, while ILD $\mathrm{PH}$ and $\mathrm{PAH}$ had lower prevalences $(7.5 \%, 6.1 \%)$. At the end of follow up, $32 \%$ of SSc pts had ILD, $13 \%$ ILD-PH and $7 \%$ PAH. ILD and ILD $\mathrm{PH}$ were more frequent in the diffuse form $(47 \%, 12 \%)$, while $\mathrm{PAH}$ did not differ between subforms. Significant differences in baseline characteristics between PAH vs. ILD-PH vs. ILD were found for age (62, 59, 54 years), sex (males: 15\%, 22\%, 24\%) and smoking prevalence (non-smok ers $49 \%, 63 \%, 57 \%$ ). Mean DLCO and FVC were $56 \% / 93 \%$ for PAH, $49 \% / 78 \%$ for ILD-PH and $56 \% / 81 \%$ for ILD. Significant decreases for DLCO $(\geq 15 \%)$ and FVC $(\geq 10 \%)$ were found in $45 \% / 26 \%$ in $\mathrm{PAH}, 45 \% /$ $26 \%$ for ILD-PH and $36 \% / 16 \%$ in ILD. All-cause mortality was $8.1 \%$ for the total cohort and differed significantly between patients without pulmo nary involvement (4\%), ILD (7.8\%), PAH (14.2\%), and ILD-PH (21\%, $\mathrm{p}<0.001$ ).

Conclusion: ILD is the most prevalent pulmonary involvement in SSc, while PH-ILD is associated with the most detrimental survival. Significant differences in baseline characteristics of types of pulmonary SSc involvement may help to identify patients at risk in the future.

Disclosure of Interests: : Michael Kreuter Grant/research support from: Boehringer Ingelheim, Roche Pharma, Consultant for: Boehringer Ingelheim, Roche Pharma, Speakers bureau: Boehringer Ingelheim, Roche Pharma, Francesco Bonella Consultant for: Boehringer Ingelheim, Roche Pharma, Speakers bureau: Boehringer Ingelheim, Roche Pharma, Ulf Müller-Ladner Grant/research support from: Projekt supported by an unrestricted educational grant from Celgene $\mathrm{GmbH}$., Elise Siegert Shareholder of: Astra Zeneca, Grant/research support from: Actelion, Consultant for: AEC Partners, Speakers bureau: Actelion, Norsk, Jörg Henes: None declared, Gabriela Riemekasten Consultant for: Chugai, F. Hoffmann-La Roche, Speakers bureau: Chugai, F. Hoffmann-La Roche, Norbert Blank Grant/research support from: SOBI and Novartis, Speakers bureau: Novartis and SOBI, Marc Schmalzing Grant/research support from: Pfizer, Chugai, MSD, Janssen-Cilag, BMS, Celgene, UCB, Consultant for: Abbvie, Chugai, Genzyme, Hexal/Sandoz, MSD, Novartis, Roche, Sanofi Pasteur Speakers bureau: Actelion, Baxalta/Shire, BMS, Celgene, Chugai, Janssen-Cilag, MSD, Novartis, Pfizer, Roche, UCB, Ina Koetter: None declared, Claudia Guenther Grant/research support from: Pfizer, Novartis, Employee of: 20 years ago, Novartis, Speakers bureau: Celtis, Tim Schmeiser Speakers bureau: Actelion, UCB, Pfizer, Gabriele Zeidler Grant/research support from:

- MSD Sharp \& Dohme GmbH

- Actelion Pharmaceuticals Deutschland GmbH

- Roche Pharma AG

- AbbVie Deutschland GmbH Co. KG

- Pfizer Pharma GmbH

- Lilly Deutschland GmbH

- Celgene $\mathrm{GmbH}$

Bristol-Myers Squibb

UCB Pharma GmbH/UCB GmbH, Speakers bureau:

- MSD Sharp \& Dohme GmbH

- Actelion Pharmaceuticals Deutschland $\mathrm{GmbH}$

- Roche Pharma AG

- AbbVie Deutschland GmbH Co. KG

- Pfizer Pharma GmbH

- Lilly Deutschland GmbH

- Celgene $\mathrm{GmbH}$

\section{Bristol-Myers Squibb}

UCB Pharma GmbH/UCB GmbH, Alexander Kreuter Speakers bureau: Actelion Pharma, MSD, AbbVie, InfektioPharm, Margitta Worm: None declared, Laura Susok: None declared, Aaron Juche: None declared, Christiane Pfeiffer: None declared, Cord Sunderkoetter: None declared Donja Homayoon: None declared, Kathrin Kuhr: None declared, HannsMartin Lorenz: None declared, Pia Moinzadeh Speakers bureau: Actelion, Nicolas Hunzelmann Speakers bureau: Boehringer Ingelheim, Actelion, Pfizer, Roche

DOI: 10.1136/annrheumdis-2019-eular.1775 